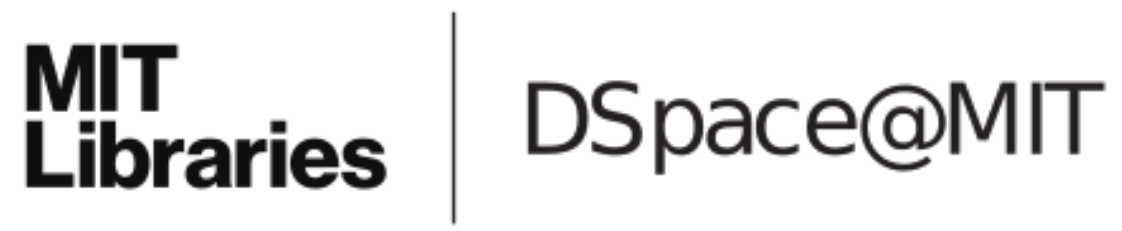

MIT Open Access Articles

Tuning up T-cell receptors

The MIT Faculty has made this article openly available. Please share how this access benefits you. Your story matters.

Citation: Rappazzo, C Garrett and Michael E Birnbaum. "Tuning up T-Cell Receptors." Nature Biotechnology 35, 12 (November 2017): 1145-1146 @ 2018 Nature Publishing Group

As Published: https://doi.org/10.1038/nbt.4009

Publisher: Nature Publishing Group

Persistent URL: http://hdl.handle.net/1721.1/119217

Version: Author's final manuscript: final author's manuscript post peer review, without publisher's formatting or copy editing

Terms of use: Creative Commons Attribution-Noncommercial-Share Alike 


\section{Tuning up T-cell receptors}

T-cell receptors with higher affinity for tumor antigens are discovered by harnessing $\mathrm{T}$ cell development.

C. Garrett Rappazzo and Michael E. Birnbaum

Dept. of Biological Engineering, Koch Institute for Integrative Cancer Research, Massachusetts Institute of Technology

Cambridge, MA 02139

Correspondence: $\underline{\text { mbirnb@mit.edu }}$

When developing new therapies, borrowing from nature has an undeniable appeal. Solutions honed by millions of years of evolution are often better starting points than what scientists can come up with from scratch. This is the principle behind cancer immunotherapies based on engineered $\mathrm{T}$ cell receptors (TCRs). Natural tumor-reactive TCRs are identified, molecularly modified to improve their function, and introduced into a patient's T cells ${ }^{1}$. But optimizing TCRs is still an imperfect technology, and the resulting $\mathrm{T}$ cells often have suboptimal activity against tumor cells and the potential for crossreactivity against normal cells. In this issue, Schmitt et al. ${ }^{2}$ present a system that borrows from the natural process of T cell development to create better TCRs for cancer immunotherapies.

TCR-engineered T cells have several advantages over other immunotherapies. Unlike checkpoint blockade, which can nonspecifically unleash all T cells, they boost the immune response specifically against the tumor and do not require that the patient have pre-existing tumor-reactive $T$ cells. And unlike T cells engineered to express chimeric antigen receptors (synthetic fusions of an extracellular antigen-binding domain and an intracellular T-cell-signaling domain), TCR-engineered T cells are not limited to targeting cell-surface proteins because they interact with peptides that are derived from intracellular and extracellular proteins and displayed on the cell surface by the major histocompatibility complex (MHC). 
Although TCR-engineered T cells have shown promise in pre-clinical and clinical studies ${ }^{1,3}$, they have not yet joined the pantheon of commonly used immunotherapies. The chief technical challenge has been to increase the anti-tumor reactivity of engineered TCRs without introducing deleterious reactivity against healthy tissue. TCRs generally bind their peptide-MHC (pMHC) ligands with low affinity ${ }^{4}$, and tumor-specific $T$ cells trend toward the lower end of the affinity range owing to the pruning of self-reactive T cells during development ${ }^{5}$. Researchers have succeeded in increasing TCR affinity through by mutagenesis of the pMHC-contacting CDR loops, much as is done for antibodies. However, this approach can inadvertently create TCRs that cross-react against normal tissues, as highlighted by a recent case in which engineered TCR T cells specific to a melanoma antigen attacked cardiac tissue ${ }^{8}$. A second technical challenge is that TCRs can be finicky and laborious to manipulate, and it is not feasible to systematically explore the full combinatorial space of all binding loop sequences and lengths $\mathrm{s}^{6,7}$.

To overcome these limitations, Schmitt et al. ${ }^{2}$ sought to coax T cells themselves to do the work of TCR diversification and selection by exploiting a quirk of T cell development. Conventional $\alpha \beta$ T cells, which comprise the large majority of a person's T cell compartment, recombine their TCR $\alpha$ and $\beta$ chains in discrete steps: TCR $\beta$ followed by TCRa. If a developing T cell successfully recombines its beta chain, it receives weak functional signals that prompt progression to alpha-chain recombination and functional maturity ${ }^{9}$. However, if it receives an unexpectedly strong signal during early development, the cell responds as if it has recombined a TCR using the alternate $y$ and $\delta$ chains, and is diverted to an alternative $\delta^{\mathrm{T}} \mathrm{T}$ cell-like developmental pathway ${ }^{10}$.

Schmitt et al. ${ }^{2}$ take advantage of the fact that triggering the $ү \delta$ pathway-and the associated strong signaling via the TCR—can be tracked with cell-surface markers. Thus, T cells that carry a fully formed $\alpha \beta$ TCR and signal at an earlier-than-expected stage of T cell development after pMHC engagement can be readily isolated. The authors harness this process to select for strongly reactive, tumor-antigen-specific TCRs (Fig. 1). They introduce a recombined TCRa chain derived from a previously validated tumor-specific TCR into developing T cells and allow the cells to mature on feeder 
cells in the presence of the tumor antigen. Cells that show a strong TCR signal during TCR rearrangement are sorted, and their TCR $\beta$ chains are sequenced and tested to identify those that confer higher affinity than the parental one.

The authors demonstrate their method with a mouse anti-tumor TCR both in vitro and in vivo and with a human anti-tumor TCR in vitro using T cell progenitors. They find that the T cells do indeed recombine TCR $\beta$ chains specific for the intended tumor antigens. As hoped, the isolated T cells contain a diverse collection of TCR V $\beta$ regions and a collection of peptide-binding CDR3 loops that are diversified in both sequence and length. The newly generated TCR $\beta$ chains recognize the intended antigen, and some bind their antigens with higher affinity than the parental chain does and signal more potently.

When vetting a new TCR for clinical development, a chief concern is unwanted recognition of healthy cells expressing non-target antigens that enable the TCR to cross-react. This a particular challenge because each TCR has its own cross-reactivity profile, and even extensive vetting against cell lines derived from multiple organs cannot rule out a negative interaction in patients. In principle, optimizing a TCR for better function through a native T cell pathway while simultaneously conserving most of the TCR interface should mitigate potential cross-reactivity. Indeed, when tested for function, the newly identified TCRs show no signs of unwanted self-reactivity in a mouse model.

With the principle demonstrated, there are some considerations in moving the approach forward. First, the system optimizes only TCR $\beta$ chains, which account for half of the antigen-binding interface ${ }^{4}$, and reliance on the $\mathrm{V}(\mathrm{D}) \mathrm{J}$ recombination machinery imposes biases on the peptidecontacting junctions, limiting diversity. These issues may explain why the observed affinity gains were modest. Additionally, it remains to be seen whether the improvements observed in vitro translate into improved anti-tumor activity in vivo. Finally, while modifying only the peptide-binding CDR3 loops should help prevent peptide-independent reactivity, and limiting TCR selection to beta chains with high germline-encoded similarity to the parental chain should help limit off-target reactivity, any improved TCR will have to undergo extensive vetting for cross-reactivity on the path to clinical development. 
These caveats aside, the method of Schmitt et al. ${ }^{2}$ could prove important for future TCR engineering efforts. In relying on T cell development, it should be applicable to any TCR. It also eliminates much of the effort and TCR-specific optimizations of other methods. Having been generated by the natural diversification and signaling machinery, the resulting TCRs already pass several of the requirements for function and specificity needed for therapeutic applications.

1. Fesnak, A. D., June, C. H. \& Levine, B. L. Engineered T cells: the promise and challenges of cancer immunotherapy. Nat Rev Cancer 16, 566-581 (2016).

2. Schmitt, T. M. et al. Generation of high affinity TCRs with diverse CDR3 regions by antigendriven differentiation of progenitor T cells in vitro. Nat. Biotechnol. (2017).

3. Morgan, R. A. et al. Cancer regression in patients after transfer of genetically engineered lymphocytes. Science 314, 126-129 (2006).

4. Rossjohn, J. et al. T cell antigen receptor recognition of antigen-presenting molecules. Annu. Rev. Immunol. 33, 169-200 (2015).

5. Aleksic, M. et al. Different affinity windows for virus and cancer-specific T-cell receptors: implications for therapeutic strategies. Eur. J. Immunol. 42, 3174-3179 (2012).

6. Smith, S. N., Harris, D. T. \& Kranz, D. M. T Cell Receptor Engineering and Analysis Using the Yeast Display Platform. Methods Mol. Biol. 1319, 95-141 (2015).

7. Li, Y. et al. Directed evolution of human T-cell receptors with picomolar affinities by phage display. Nat. Biotechnol. 23, 349-354 (2005).

8. Cameron, B. J. et al. Identification of a Titin-derived HLA-A1-presented peptide as a crossreactive target for engineered MAGE A3-directed T cells. Sci Trans/ Med 5, 197ra103-197ra103 (2013). 
9. Boehmer, von, H. et al. Pleiotropic changes controlled by the pre-T-cell receptor. Curr. Opin. Immunol. 11, 135-142 (1999).

10. Pennington, D. J., Silva-Santos, B. \& Hayday, A. C. Gammadelta T cell development--having the strength to get there. Curr. Opin. Immunol. 17, 108-115 (2005).

Figure 1: Overview of the T cell-based TCR optimization strategy. (a) During T cell development (top), the pre-TCR $\alpha$ and recombined TCR $\beta$ weakly signal during TCR $\beta$ chain selection to promote differentiation to a mature, naturally occurring CD4 or CD8 T cell. If there is a strong TCR signal during TCR $\beta$ chain selection, caused by a pre-recombined TCR $\alpha$ chain, T cells differentiate instead into a trackable, undeveloped phenotype (bottom). (b) TCRs that induce a strong signal can be isolated, transduced into mature T cells, and tested for function. The TCRs isolated in this way have optimized TCR $\beta$ chains that show increased affinity for antigen. 